\title{
AN EFFICIENT AUTOMOTIVE COLLISION AVOIDANCE SYSTEM FOR INDIAN TRAFFIC CONDITIONS
}

\author{
Vyom Bhushan', Sugandh Goswami \\ ${ }^{1}$ Automobile Engineering, IOK COE, Pune, Maharashtra, India \\ ${ }^{2}$ E\&TC Engineering, IOK COE, Pune, Maharashtra, India
}

\begin{abstract}
The execution of a wide-ranging accident cautioning system is being visualised by ACAS Program, which is proficient in perceiving and cautioning the driver of potential hazard conditions forward, on the side, and rear sections of the vehicle. The structure would use: (a) long array sensor or visual devices to identify probable hazards in front of the vehicle, (2) short range sensors to caution the driver of nearby objects while changing traffic lanes or backing up, and (3) a track exposure arrangement to alert the motorist when the vehicle deviates from the intended traffic lane. Accident Prevention organisms, as a consequent step to collision mitigation, are one of the Great challenges in the area of active safety for road vehicles. The task of a collision avoidance system is to track objects of potential collision threat and govern any action to evade or diminish a crash. The main focus is how to make decisions based on ambiguous evaluations and in the presence of numerous hurdles. The first step in CA (Collision Avoidance) systems for automotive applications is adaptive cruise control (ACC). The ability of the car to protect its passengers is sometimes called crashworthiness. Generally, a CM (Collision Mitigation) system tries to reduce the severity of the accident as much as possible under some constraints. The situation may be observed with detector sensors, laser radar, vision sensors, ultrasonic sensors, GPS sensors and inter-vehicle communication. FCM (Forward Collision Mitigation) systems mainly try to avoid or mitigate frontal collisions. The CA decision is based on the current position estimate of the host vehicle and those of other objects. The focus is towards the method for determining the threat of a collision given that the state of other objects is known. Two collision mitigation by braking systems are considered for study. One system uses the probability of collision, to decide when to perform braking interventions. The other system has a multiple obstacle decision. The scenarios that are calculated in this segment are primarily those where a CMbB has a large potential of significantly reducing the collision speed. Therefore, the test results of main interest is those between 0 and $70 \mathrm{~km} / \mathrm{h}$ also for simulations. Hence, this ACAS Program detects the potential hazards, warns the driver and takes action to avoid or mitigate a collision.
\end{abstract}

Keywords: Collision Warning, Decision Making, Collision Mitigation Braking, Tracking Sensors

\section{INTRODUCTION}

Eluding impacts is a critical issue in most transportation systems. The task of any collision avoidance system is ultimately to avoid two or more objects from colliding. ${ }^{[1]}$

The ACAS Program visualizes the application of a complete accident warning system, which is capable of detecting and cautioning the motorist of probable risk conditions in the forward, side, and rear regions of the vehicle. The imminent collision can be reduced by collision avoidance system. Collisions are avoided through CA systems either by warning the driver about the collision or by performing an autonomous avoidance manoeuvre. There are some other actions might be taken for mitigating collision consequences in an automotive application such as pretension of seat belts and inflation of airbags. Any action performed by CA system is said to be an intervention.

Adaptive cruise control (ACC) is the first step for automotive application in CA system. The speed to any inpath vehicle is adapted by ACC system. This system only exerts limit retardation; there are some other systems also which warn the driver when this acceleration is in sufficient for avoiding the collision. As per the requirement, driver can switch on and off ACC system. Generally, these systems are automatically disengaged at lower speed (below $40 \mathrm{~km} / \mathrm{h}$ ). Now, here is the next step in automotive CA which introduces the systems capable of performing autonomous braking and/or warning the driver if the collision is imminent and the system always remains active.

\section{LITERATURE REVIEW}

The Automotive Collision Avoidance Systems Program was originally set up to be a two-year program with activities starting in January 1995. The events were carried out by a consortium made up of government agencies as well as, industrial and academic participants of U.S. ${ }^{[5]}$

Jansson $^{[1]}$, Jonas in his thesis, 'Collision Avoidance Theory with Application to Automotive Collision Mitigation' presented the concept for following and choice making in crash evasion structure.

Kochenderfer $^{[2]}$, Mykel J., Chryssanthacopoulos, James P. and Weibel, Roland E., presented a paper titled, 'A New Approach for Designing Safer Collision Avoidance Systems'. This theory defined the overall methodology and 
deliberated the anticipated impact on development, safety and operation.

Ramesh $^{[3]}$, S., Ranjan Ravi, Mukherjee Ranjeet and Chaudhuri Swarnali presented a theory in an international periodical titled, 'Vehicle Collision Avoidance System Using Wireless Sensor Networks' for the improvement of new processing procedures to prevention of impacting automobiles.

Varghese ${ }^{[4]}$ Babu, Jacob Renju Thomas, Kamar Fajas and Saifudeen Rizwan Ali, presented a paper in an international journal in 2014, titled 'Collision Avoidance System in Heavy Traffic and Blind Spot Assist Using Ultrasonic Sensor' to project a collision avoidance system which is trustworthy for motorists in heavy traffic where the speed is normally below $20 \mathrm{~km} / \mathrm{hr}$.

Final Report ${ }^{[5]}$ on 'Automotive Collision Avoidance System (ACAS) Program' by U. S. Department of Transportation, National Highway Traffic Safety Administration (NHTSA) of August 2000, focussed on devices, human factors, systems such as route assessment, in-path destination choice and danger calculation.

Basma ${ }^{[6]}$ Fadi and Refai, Hazem H. offered a report in December 2009 on, 'Collision Avoidance System at Intersections' in University of Oklahoma, which approves the aptitude of the structure to deliver a cautionary indicator in high likelihood collision circumstances.

Bella ${ }^{[7]}$ Francesco and Russo Roberta, presented a paper on, 'A Collision Warning System for rear-end collision: a driving simulator study', studying the driver's conduct during car-following tactics.

Hafner ${ }^{[8]}$, M. R., Cunningham, D., Caminiti, L. and Vecchio, D. Del, presented a paper titled, 'Automated Vehicle-toVehicle Collision Avoidance at Intersections' in which they delivered trial consequences for an automatic control system proficient in averting impacts between two vehicles at an intersection.

\section{AUTOMOTIVE SAFETY}

Despite the improved crashworthiness of modern cars, it is clear that there is a strong requirement to reduce traffic related injuries. Having evolved rapidly, existing passive safety technologies have become quite mature. Therefore, a significant reduction of injuries by passive safety technologies becomes technically infeasible as well as too costly. In recent years, people in the automotive industry have increased their efforts in trying to find other ways of reducing the number of people injured in traffic. The $\mathrm{CM}$ system uses sensors to observe the environment directly in front of the host vehicle. Based on information from the forward-looking sensors, a decision can be made to deploy countermeasures to avoid imminent frontal collisions. Typical interventions are warning signals (audible, visible or tactile), braking and steering.

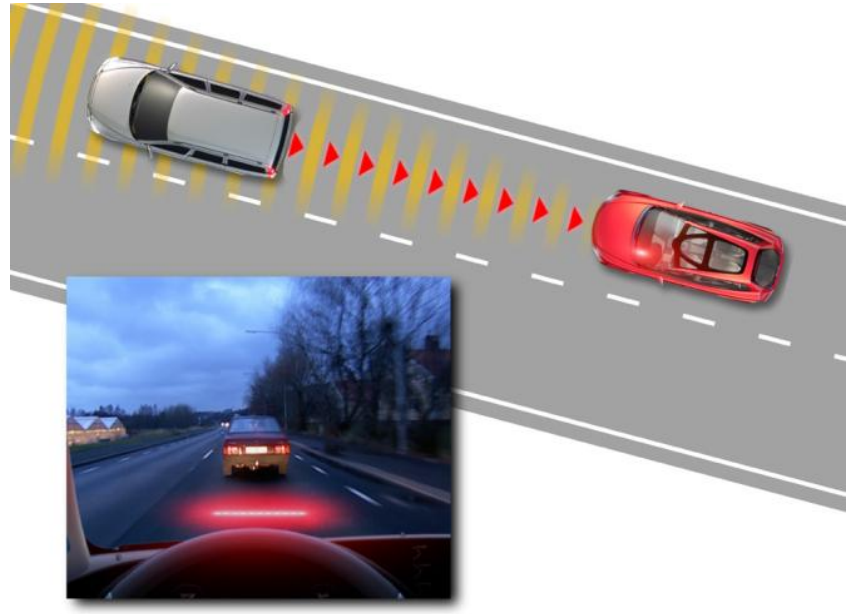

Fig-1: Example of a CM System ${ }^{[1]}$

The device uses radar to measure space and speed comparative to the vehicle in front. In the event of probable ramming, the device informs the motorist by a blinking red light projected on the windshield.

\subsection{Passive Safety}

When an automobile's inactive security properties are measured, it states the talent of the car to guard its travellers from damages in an accident; then it is generally termed as crashworthiness. In this paper, the crashworthiness of a car is demarcated as its ability to safeguard passengers from injury from the moment the collision happens and subsequently. One way to attain decent reflexive safety is to enhance the assembly of the car's body in such a way that it grips crash energy whilst keeping the crash pulse (instantaneous acceleration) experienced by the passengers as low as possible. Selected arrangements that help to safeguard the passengers in a collision are seat belts, airbags, belt pretensions, and belt load regulators. The interior of the automobile should also be planned in such a way that panels, switches, etc. cause minimal damage. To accomplish worthy reflexive security properties, certain spaces of the car's plan must be well-thought-out.

\subsection{Active Safety}

Active safety includes a number of different characteristics of the car. Here, they are distributed into three sets: preventive, dynamic and collision mitigation.

\subsubsection{Preventive}

The precautionary feature of dynamic safety is about the motorist seeing dangers. If dangers are identified in the initial stages, the driver can take protective action before the condition gets out of control. ${ }^{[1]}$

\subsubsection{Dynamic}

From a safety perspective, a car should be designed to have safe handling and ride characteristics. It should be easy for the driver to keep control of the vehicle in all road conditions, in any traffic situation and during all types of manoeuvres. ${ }^{[1]}$ 


\subsubsection{Collision Mitigation}

CM arrangements are granted certain sensitivity of the setting and situation surrounding the vehicle. Based on this sensitivity, the system takes steps to avoid or mitigate imminent collisions. It must be pointed out that these systems are often called Collision Avoidance systems. The environment may be perceived with radar sensors, laser radar, vision sensors, ultrasonic sensors, GPS sensors and inter-vehicle communication. ${ }^{[1]}$

\subsection{Forward Collision Mitigation}

Forward CM systems mainly try to avoid or mitigate frontal collisions. Forward collision warning systems are referred to as FCW structures or Forward Collision Mitigation by slowing systems as $\mathrm{CMbB}$ systems.

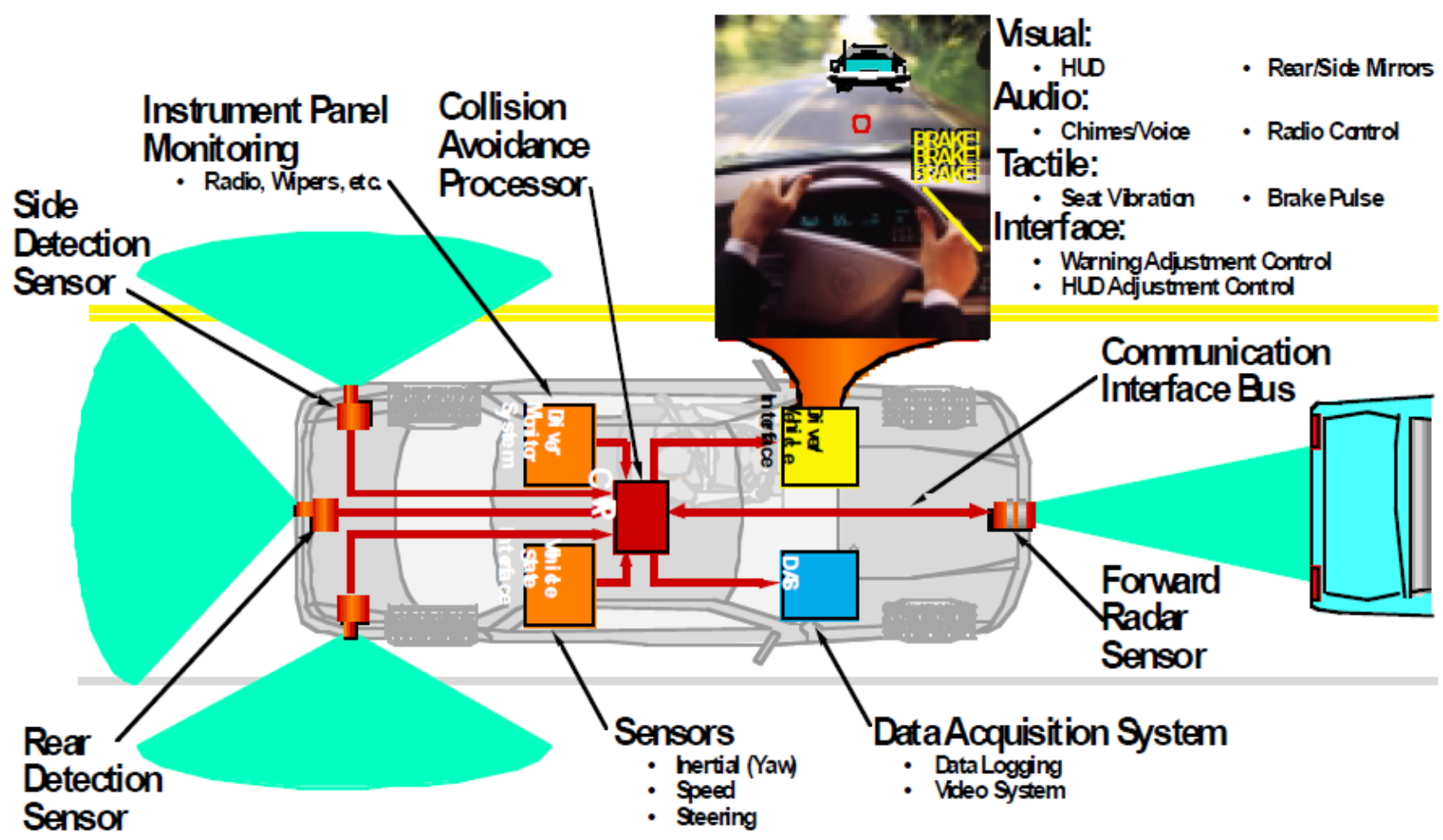

Fig-2: Collision Warning Vehicle Mechanization ${ }^{[5]}$

The timing in FCW and CMbB systems is almost different. In a FCW system, driver reaction time must be taken into account. In [1] it is stated that $85 \%$ of all drivers are able to react to a warning within 1.18 seconds. In a FCW system several faulty interventions are tolerated, whereas in CMbB systems faulty interventions are not allowed practically.

\section{INTERSECTION COLLISION WARNING}

ICW utilizes wireless sensor networks (WSN) for detection and transfer of warning information to the driver to avoid accidents. The system is set up into intersection roadways and supports real time prevention by monitoring approaching movement and giving an alert to motorists when there is a high probability of collision.

\subsection{System Overview}

The foremost purpose of ICW is to avoid collision at rural intersections. The system is based exclusively on infrastructure communication and is set up into roadways around the intersection.

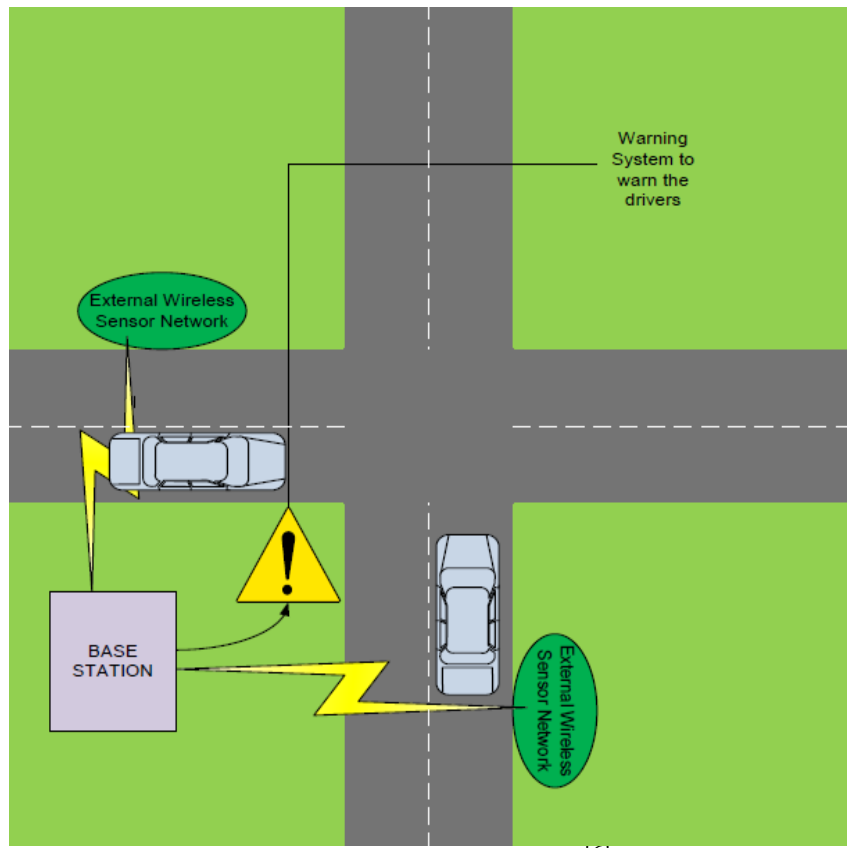

Fig-3: Overview of $\mathrm{ICW}^{[6]}$ 
Fig-3 depicts a high-level overview of the entire system.

The system is comprised of the following:

\subsubsection{External Sensor Nodes}

Gather automobile statistics transiting through the radar. ${ }^{[6]}$

\subsubsection{Base Station (BS)}

Situated at the joint of every crossing to study data; collects evidence from exterior sensor nodules wirelessly. ${ }^{[6]}$

\subsubsection{Warning System}

Situated at leading and insignificant motorway intersections to trigger a cautionary indicator after BS has analysed data and determine the possibility of a collision. ${ }^{[6]}$

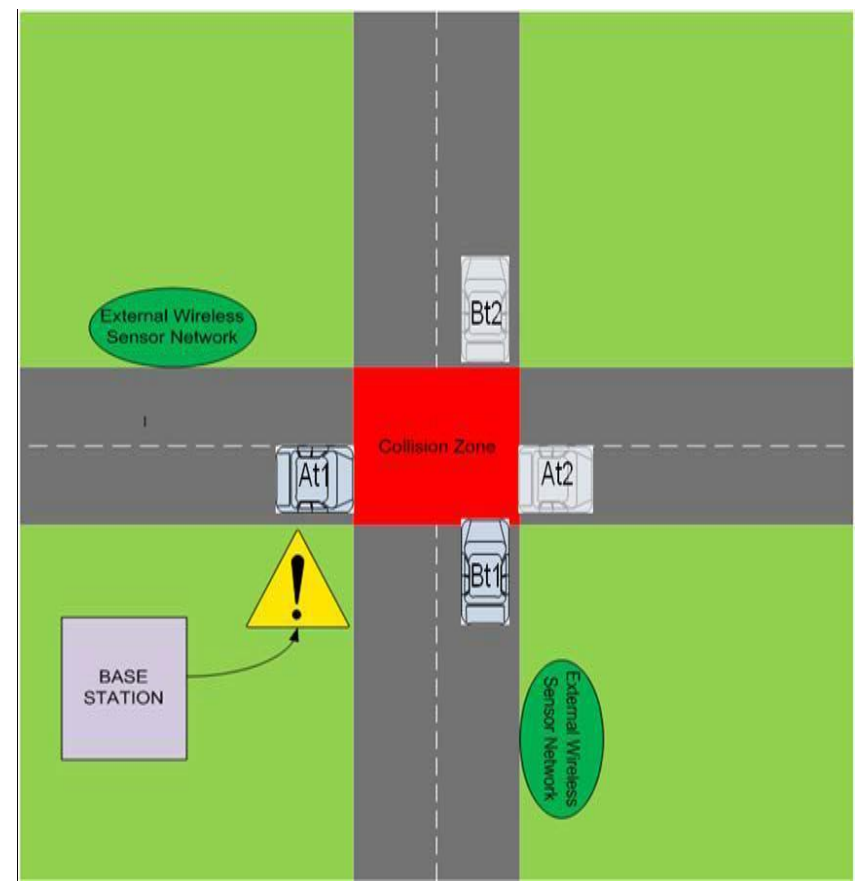

Fig-4: Representation of two vehicles entering and exiting the intersection ${ }^{[6]}$

\section{AUTOMOTIVE TRACKING SENSORS}

The sensors used to track objects and monitors, the movement of the host vehicle as well as the movement of the surrounding objects to predict collisions are known as 'tracking sensors'. In automotive applications, cost of the sensor is a major factor.

\subsection{Radar Sensor}

Radio detection and ranging (Radar) is the most commonly used tracking sensor in automotive detection applications. The important measurements provided by the radar sensor are range $(r)$, range rate $\left(\mathrm{r}^{\circ}\right)$, azimuth angle $(\phi)$, and elevation angle.

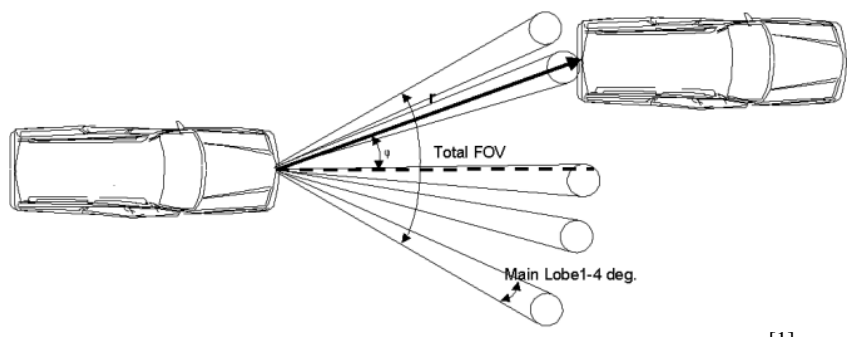

Fig-5:Measurements provided by the Radar Sensor ${ }^{[1]}$

The radar sensor is an active sensor as it emits electromagnetic radiation for illuminating targets. Same antenna emits and receives the radar waves, and the sensor constantly switches between the sending and receiving mode. Long range automotive radars have the frequencies in the range of 76-77 GHz. Other automotive radar sensors are operated in the $5 \mathrm{GHz}$ and $24 \mathrm{GHz}$ regions.

\subsection{Lidar Sensor}

Laser radar (lidar) is similar to a mm-radar in its basic function. Lidar provides range, range rate, azimuth and elevation measurements. Lidar is operated in the infrared frequency region having wavelengths around $850 \mathrm{~nm}$.

\subsection{Vision Systems For Obstacle Recognition}

Vision systems use one or several cameras together through a microprocessor for performing image processing. As they operate in the visible light region, they have the capabilities similar to that of our eyes.

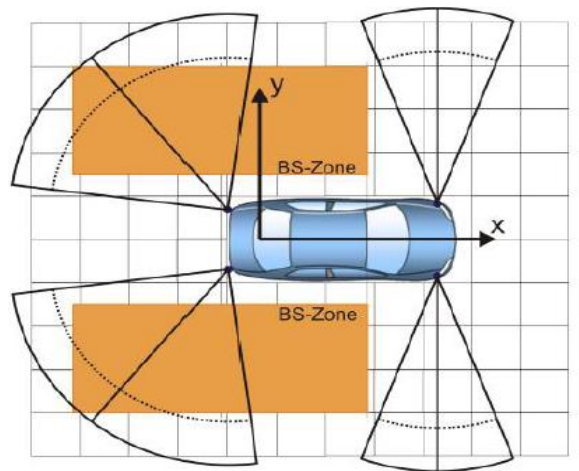

Fig-6:The outline of a host car (with blind spot assist) with sensor cones ${ }^{[4]}$

The two main types of system are:

\subsubsection{Single Camera Systems}

Using one or the other monochrome or a colour camera; primary usage in motorized applications for single camera systems is to observe the lane markings in lane-keeping aid systems. ${ }^{[1]}$

\subsubsection{Stereo Camera Systems}

A high-fidelity camera system provides a 3D appearance by uniting the pictures from two (or more) cameras. In such a system, distance can be measured through triangulation. Due to the $3 \mathrm{D}$ evidence, difficulty recognition is easy. ${ }^{[1]}$ 


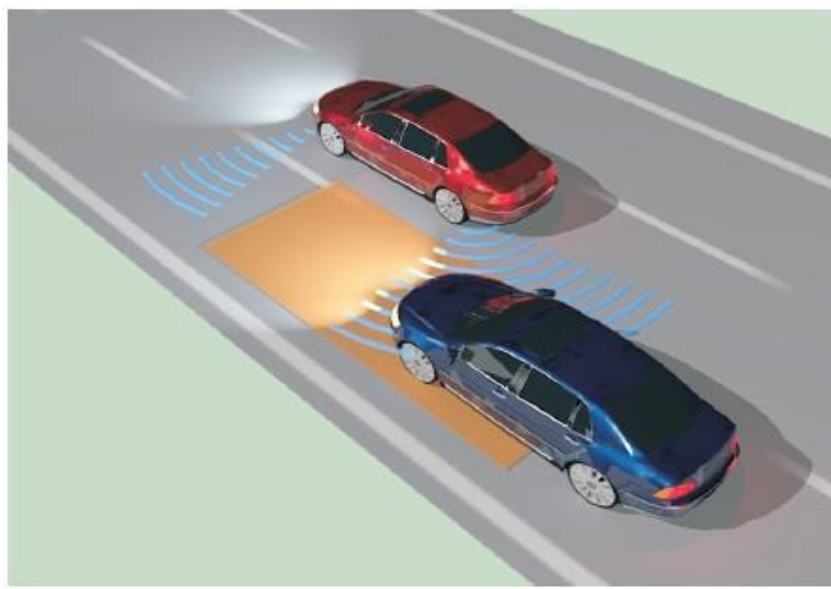

Fig-7:A vehicle approaching into the blind spot of a host vehicle $^{[4]}$

\subsection{Infrared Vision Sensor}

In automotive systems, IR cameras are introduced for night vision systems. Night vision systems enhance the driver's perceptive abilities (Fig-8). The sensors are most sensitive to wavelengths corresponding to the normal body temperature of humans and large animals. IR cameras are not used in automotive collision mitigation (CM) systems but they are much helpful to the driver for night vision. The IR sensors have a unique ability of measuring the temperature of objects.

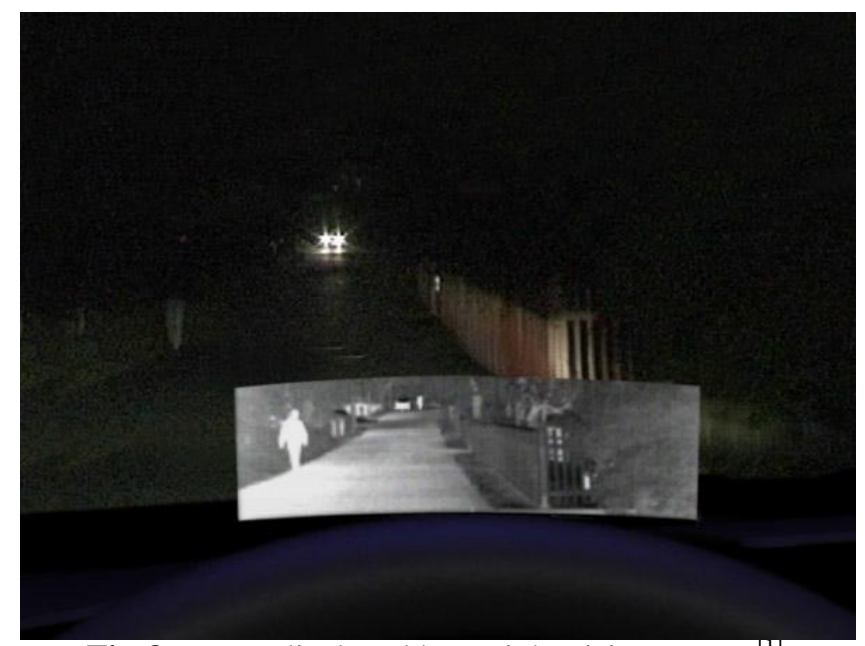

Fig-8: Image displayed by a night vision system ${ }^{[1]}$

IR cameras could be so useful in classifying objects and providing accurate angle measurements. For example, in certain automobiles, the exhaust pipe is noticeable and easily observed in a heat sensitive camera. Humans and animals are also often easily detected in an IR-image.

\subsection{Inter-Vehicle Communication}

Most of the vehicles are generally equipped with navigation systems consisting of GPS (Global Positioning System) receivers and digital maps. If any car is equipped with a communication system it is possible to transmit the navigation data to other vehicles. This data can be used by the tracking system of the other vehicle.

\section{DECISION MAKING}

The collision avoidance (CA) system is mainly used for avoiding or mitigating collisions by self-directed action or warning. Decision making is defined as the task to decide an action. The CA decision depends on the current location estimate of the host vehicle and other objects. The tracking system provides the estimates. The position estimates are uncertain due to the measurement and process noise. The state estimate uncertainty is not considered.

\subsection{Collision Avoidance Decision Strategy}

The selection of CA intervention strategy has the problem of its contradictory properties. Five different states can be defined for analysing possible strategies.

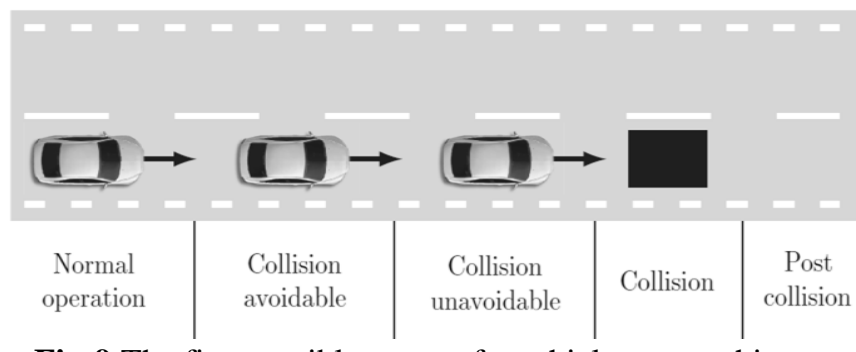

Fig-9:The five possible states of a vehicle approaching a stationary obstacle. ${ }^{[1]}$

These states are represented for an automotive scenario where a vehicle is approaching a stationary obstacle. Each state is briefly described below:

\subsubsection{Normal Operation}

In this condition, there is no impending danger or hazard of a collision. ${ }^{[1]}$

\subsubsection{Collision Avoidable}

When there is a threat to the vehicle. A non-negligible risk exists that a collision will occur. In this state, there is a possibility to avoid the imminent collision by an appropriate avoidance maneuver. Typically, this state is perceived by a human as dangerous. It is normally in this state that collision warning systems are activated. A system that intends to evade collision has to work in this situation. ${ }^{[1]}$

\subsubsection{Collision Unavoidable}

In this state, a collision is imminent, and cannot be avoided by any manoeuvres. Even though the collision cannot be avoided, it might still be possible to considerably reduce its severity by reducing the collision speed and by taking other mitigating actions. ${ }^{[1]}$

\subsubsection{Collision}

This is the state when a collision occurs. ${ }^{[1]}$ 


\subsubsection{Post Collision}

The state when a collision has occurred is called post collision. Actions to avoid secondary collisions can be considered, if the CA system is operational even after collision. Often it is possible to go directly from the normal operating state to the collision unavoidable state because information of the surrounding environment is limited. A multiple collision on a free-way is a common example of such an event. In such case, a following vehicle is in the normal operating state until the lead vehicle suddenly collides. In dense traffic and small time gap to the lead vehicle, the collision at that moment becomes unavoidable. In Fig-10 and Fig-11, two examples of such situations are illustrated.

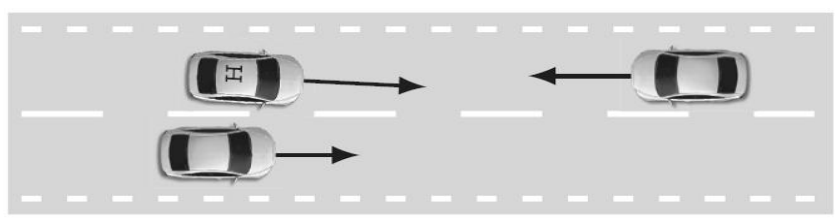

Fig-10:Overtaking Situation ${ }^{[1]}$

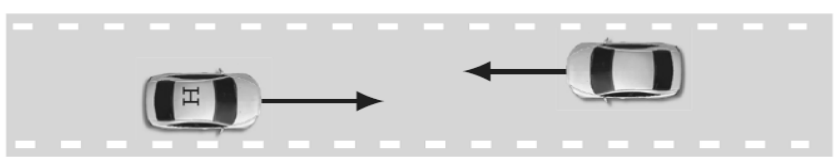

Fig-11: Meeting On A Narrow Road Situation ${ }^{[1]}$

In collision warning ( $\mathrm{CW}$ ) systems, the decision boundary must be in the collision avoidable state; with sufficient interval to respond before the unavoidable accident state is reached. For an inattentive driver to be able to avoid an imminent collision, the warning normally has to come more than one second before the collision unavoidable state is entered. ${ }^{[1]}$

\section{COLLISION MITIGATION BRAKING SYSTEM}

The braking operation is assisted by CMBS as it automatically appropriate amount of braking force to help in avoiding or minimizing the chances of heating a vehicle ahead in traffic. The location of potential collision is indicated around the intersection of the paths in Fig-12 while two adjacent vehicles are approaching a four way intersection along predefined paths.

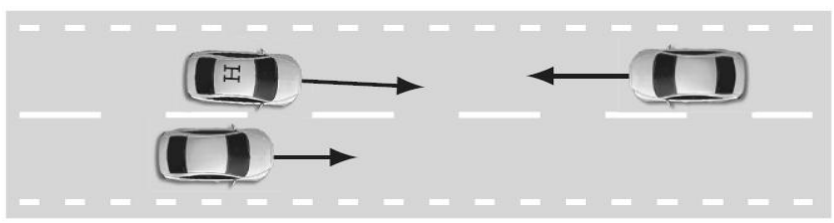

Fig-12:The location of potential collision around intersection of the paths. ${ }^{[8]}$
The CMBS consists of the following:

- A radar sensor in the front grille,

- A brake actuator in the engine compartment,

- An display on the device section,

- Seat belt e-pretensions on the front seats, and

- An on/off switch on the dashboard.

CMBS is based on the evaluation of the Time-to-collision (TTC). There is an initial phase of attention if the distance among the two vehicles surpasses the safety limit, valued with a onset of TTC equal to 3 seconds. When this threshold is surpassed, an alarm indicates the driver to follow precautionary action to avoid a crash. ${ }^{[7]}$

When the CMBS is on, ahead vehicle is constantly scanned by the radar sensor. If a possible collision with the vehicle ahead of you is sensed by the system, it responds with an audible alarm and a visual alarm. The CMBS radar sensor in the front grille is able to sense a vehicle ahead of you, when the speed of host vehicle is above $15 \mathrm{~km} / \mathrm{h}$. When your vehicle is too close to the vehicle ahead of you, a warning beep is activated by the system and it causes automatic application of the brakes and causes e-pretensions tighten the front seat belts.

For study, two CMbB systems are considered; one, which is used for deciding the time performing the braking interventions by using the collision probability. In this system, only individual object is considered and there is no consideration in relation to other objects. The second system considers multiple obstacle detection.

Both systems' aim is to reduce the speed of collision under the restriction for allowing the no faulty interventions only. Before the vehicle enters to the collision unavoidable driving state, the brakes are engaged by the system automatically. Both systems can be evaluated by two methods. Firstly by simulation, and the other by field tests with demonstrator vehicles.

\subsection{Collision Scenarios}

The scenarios where $\mathrm{CMbB}$ having large potential of significantly reducing the collision speed are mainly studied in this section. Between colliding vehicles, Angular accidents having the large angle rear end collisions having large lateral offset and frontal collisions having large relative speed are the examples of scenarios having low potential CMbB for the reduction of collision speed.

\subsubsection{Single Collision Decision Making Test}

1. General Scenarios

a) Head-on to Stationary Object

This type of scenario has been represented in Fig-9. This scenario is common in real life accidents and often it is 
found that the driver of the striking vehicle is not attentive or even falls asleep, and hits a stationary object.

b) Rear End Collision, Lead Vehicle Brakes Hard An accident between two vehicles moving in the same direction, in which the trailing vehicle collides the front vehicle's rear end with its front end is said to be a rear end collision. This scenario is shown in Fig-13.

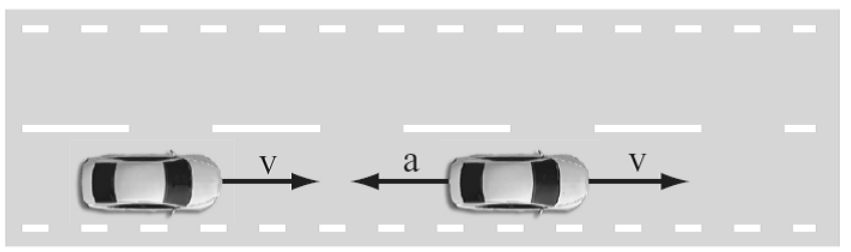

Fig-13: Rear-end collision scenario. Initially both vehicles travel with the same velocity. Suddenly the leading vehicle brakes hard, achieving a deceleration of $8 \mathrm{~m} / \mathrm{s}^{2}{ }^{[1]}$

It is found that the performance of the CMbB system for this type of accident generally varies with the following factors:

- Relative speed of the vehicles

- Headway distance

- Braking force applied by the lead vehicle

- Lateral offset

c) Cut-in Behind a Stationary Object

In this type of scenario, the host vehicle travels straight ahead, and it suddenly changes the lane which works as a stationary obstacle. The lane change manoeuvre is supported out as a sinusoid effort to the steering wheel.

The duration of the manoeuvre is $3.14 \mathrm{~s}$ for speeds below 40 $\mathrm{km} / \mathrm{h}$ and $1.57 \mathrm{~s}$ for speeds above $40 \mathrm{~km} / \mathrm{h}$. The amplitude of the input manoeuvre is tuned to give a $2 \mathrm{~m}$ lateral displacement. ${ }^{[1]}$ The scenario is represented in Fig-14.

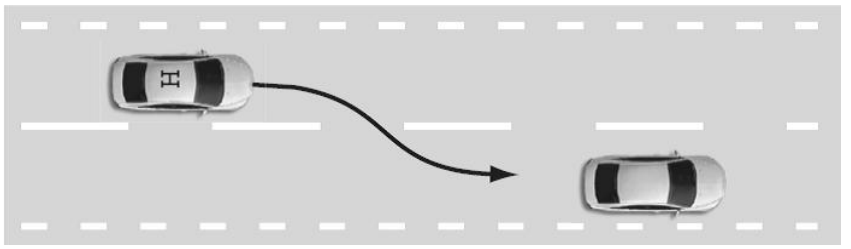

Fig-14: Cut-in scenario. The host vehicle makes a sudden lane change; in the lane that it enters there is a stationary obstacle. ${ }^{[1]}$

Generally, for most of the speeds, an average speed reduction of $5 \mathrm{~km} / \mathrm{h}$ is obtained while for low speeds, the greater reduction is achieved and there is no speed reduction for the speeds greater than $100 \mathrm{~km} / \mathrm{h}$.

d) Cut-in, in Front of the Host Vehicle

This scenario is the opposite of the previous one, where the host vehicle drives straight ahead and the other vehicle moving at a lower speed enters the host vehicle's lane in front of it. The scenario is shown in Figure 15. This scenario analyses the ability of the tracking system for tracking a sudden manoeuvre of a tracked object.

The efficiency of the CMbB system depends on the following factors:

- Relative speed between the two vehicles

- How close the lead vehicle cuts in front of host vehicle?

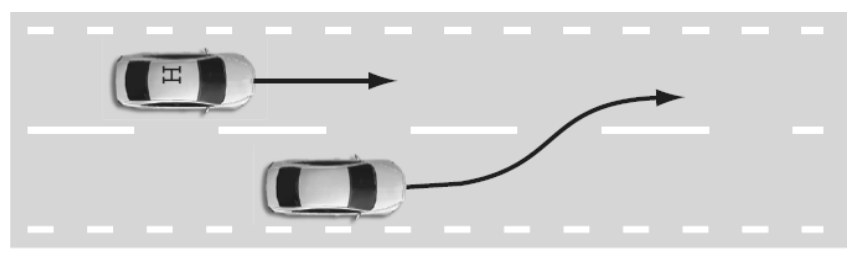

Fig-15: Cut-in scenario. The other vehicle that is travelling at a lesser speed enters the same lane as the host vehicle. ${ }^{[1]}$

Here, the relative speed is low. The other vehicle is travelling $20 \mathrm{~km} / \mathrm{h}$ slower than the host vehicle, the cut-in occurs late when the time to collision is one second. For initial host speeds up to $60 \mathrm{~km} / \mathrm{h}$, the collision speed is reduced almost $10 \mathrm{~km} / \mathrm{h}$ on the average. ${ }^{[1]}$

\section{Scenarios to Provoke Faulty Interventions}

When the host vehicle is near to the collision, the scenarios to provoke faulty interventions are needed. The $\mathrm{CMbB}$ system intervention is considered as a faulty intervention, as no collision takes place in these scenarios.

\section{a) Drive-by Scenario}

It is the special type of head-on collision scenario. In this scenario, there is large offset between the host vehicle and the obstacle for preventing the host vehicle from colliding it with the stationary object while passing it. This scenario is shown in Fig-16.

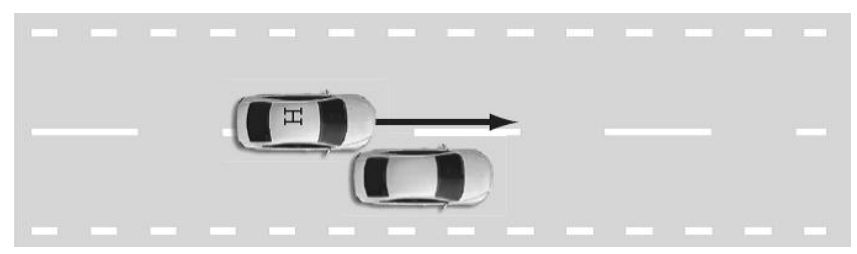

Fig-16: Drive-by scenario, the offset between the host vehicles is just large enough for the two vehicles not to collide. $^{[1]}$

It has been often noticed that measurements from the mmradar seems to be coming from an object which is moving into the host vehicle's path.

\section{b) Head-on to Stationary Object Late Avoidance}

It is that manoeuvre in which the host vehicle moves towards the stationary obstacle and the collision is prevented by steering away as late as possible This manoeuvre is represented in Fig-17. No intervention is considered for the simulated late avoidance scenarios. 

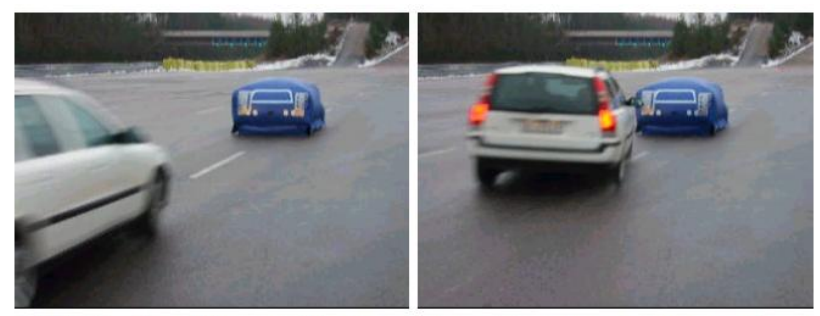

(a) Initially, the vehicle app
tionary obstacle head-on.

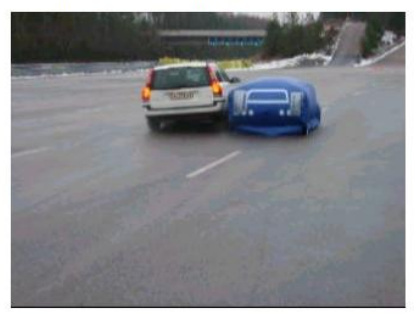
(c) The maneuver is sufficient to avoid the ob-
stacle with the smallest possible margin.

Fig-17: Late avoidance manoeuvre. ${ }^{[1]}$

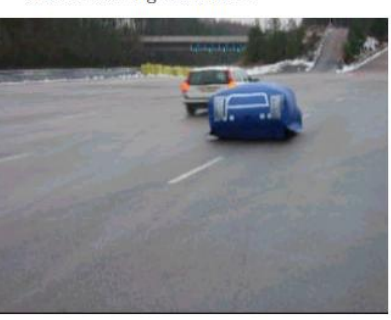

During the maneuver the driver turned the steering wheel as fast as possible. The enthis vehicle's handling capabilities.

\subsubsection{Multiple Collision Decision Making Test}

It is the head-on collision scenario with three stationary obstacles at nearly $70 \mathrm{~km} / \mathrm{h}$.

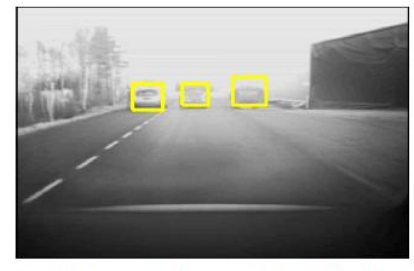

(a) Three ob
$70 \mathrm{~km} / \mathrm{h}$.

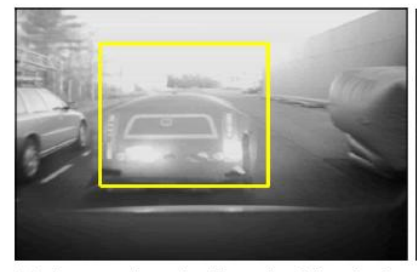

(c) At approximately $10 \mathrm{~m}$ the driver brakes to reduce the collision speed.

Fig-18: Collision scenario seen from the vision sensor's view. $^{[1]}$

The single object algorithm displays moderate threat member values if there is the requirement of high acceleration for avoiding a collision is indicated by multiple object algorithm.

\section{CONCLUSION}

Generally, a CM (Collision Mitigation) system tries to reduce the severity of the accident as much as possible under some constraints. The perception of a CM system can come from several sources. A novel real time probabilistic situation analysis approach was proposed to facilitate a collision mitigation application for vulnerable road user protection provided that a perfect environment description would exist. The distance given from the proposed example can be taken as an onset that might activate an alarm system for driver assistance in order to advise the motorist if he is in the accident prone zone. The proposed algorithm reflects the real risk perception by car user. Hence, it should lessen incorrect alarms and must help to evade a potential collision. To enterprise a virtuous accident prevention system, we must solve two issues. One is the risk estimation and the other issue, which is not being mentioned here, is regarding identifying the object. This is a matter of the sensing capabilities of the devices as well as a matter of sensor fusion. For correct decision making accurate target classification and feature extraction is imperative. A novel real time probabilistic situation analysis approach was proposed to facilitate a collision mitigation application for vulnerable road user protection provided that a perfect environment description would exist.

Furthermore, the algorithm can be generalized to fit for arbitrary objects as long as they provide information about their position, dimensions, directional velocity and the corresponding variances. Finally, relevant test scenarios and novel evaluation techniques were introduced.

Hence, this ACAS Program detects the potential hazards and warns the driver and takes action to avoid or mitigate a collision.

\section{REFERENCES}

[1]. Jansson Jonas, "Collision Avoidance Theory with Application to Automotive Collision Mitigation", Linköping Studies in Science and Technology, Dissertations No. 950, Department of Electrical Engineering, Linköping University, SE-581 83 Linköping, Sweden, 2005, ISBN 91-85299-45-6, ISSN 0345-7524, pp. 9-15, 28-32, 123-129.

[2]. Kochenderfer, Mykel J., Chryssanthacopoulos, James P. and Weibel, Roland E., "A New Approach for Designing Safer Collision Avoidance Systems”, Ninth USA/Europe Air Traffic Management Research and Development Seminar (ATM2011), Lincoln Laboratory Massachusetts Institute of Technology, Lexington, Massachusetts, USA.

[3]. Ramesh, S., Ranjan, Ravi, Mukherjee, Ranjeet and Chaudhuri, Swarnali, "Vehicle Collision Avoidance System Using Wireless Sensor Networks", International Journal of Soft Computing and Engineering (IJSCE), ISSN: 2231-2307, Volume-2, Issue-5, November 2012, pp. 302.

[4]. Varghese, Babu, Jacob, Renju Thomas, Kamar, Fajas and Saifudeen, Rizwan Ali, "Collision Avoidance System in Heavy Traffic and Blind Spot Assist Using Ultrasonic Sensor", International Journal of Computer Science and Engineering Communications (IJCSEC) ISSN:2347-8586, Volume-2, Issue-1, February 2014, pp. 95 .

[5]. U. S. Department of Transportation, Report on 'Automotive Collision Avoidance System (ACAS) Program', National Highway Traffic Safety Administration, August 2000. NHTSA Final Report: ACAS Program, 1998 (Section 3.4). 
[6]. Oklahoma Department of Transportation, Report on 'Collision Avoidance System at Intersections', by Basma, Fadi and Refai, Hazem H., University of Oklahoma, December 2009. Final Report: ICW System, 2009 (Section 4.7).

[7]. Bella, Francesco, Russo, Roberta, "A Collision Warning System for rear-end collision: a driving simulator study", International Journal of Procedia Social and Behavioral Sciences, 20, 2011, pp. 680.

[8]. Hafner, M. R., Cunningham, D., Caminiti, L. and Vecchio D. Del, "Automated Vehicle-to-Vehicle Collision Avoidance at Intersections".

\section{BIOGRAPHIES}

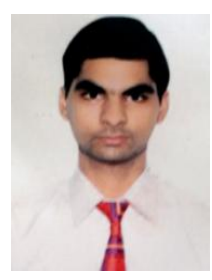

Vyom Bhushan

Final Year Student

Automobile Engineering Dept.

IOK College of Engineering

Pune-412208

India

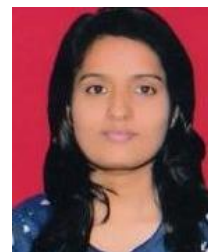

Sugandh Goswami

Final Year Student

E\&TC Engineering Dept.

IOK College of Engineering

Pune-412208

India 